\title{
Comparative study of different topologies of solar photovoltaic fed impedance-source inverter based dynamic voltage restorer
}

\author{
Miska Prasad*, Almoataz Youssef Abdelaziz** and Ashok Kumar Akella*** \\ * Department of Electrical \& Electronics Engineering, ACE Engineering College, Secunderabad India. \\ ** Faculty of Engineering \& Technology, Future University in Egypt, Cairo, Egypt. \\ *** Department of Electrical \& Electronics Engineering, NIT Jamshedpur, India. \\ *Corresponding Author: pmiska25@gmail.com
}

Submitted :24/03/2020

Revised : 19/07/2021

Accepted :01/08/2021

\begin{abstract}
All customers require quality voltage, quality current, and quality power from the utility, but usage of nonlinear loads generates voltage sag, swell, and harmonics. This work examines the solar energy based various topologies like 3-Leg, 4-Leg, and 6-Leg Dynamic Voltage Restorer (DVR) for minimizing the voltage swell. To get the most prominent power from the solar system, Perturb \& Observe $(\mathrm{P} \& \mathrm{O})$ scheme is also used. The most famous topologies are approved utilizing MATLAB/SIMULINK programming to limiting voltage swell. The gained results are comparable nonetheless even possibly better in 6-leg PV-ZSI-DVR diverged from 3-leg and 4-leg PV-ZSI based DVRs.
\end{abstract}

Keywords: Voltage quality; Renewable Energy; Perturbation \& Observation; ZSI.

\section{INTRODUCTION}

Nowadays, all customers require quality voltage, quality current and quality power from the utility, but usage of nonlinear loads generates voltage sag, swell and harmonics (Saeed \& Raheef 2021; Haytham et al., 2020; Amam et. al., 2020). To improve the quality of the power system, various topologies of D-FACTS devices like three-phase three-leg, three-phase four-leg, and three-phase six-leg based dynamic voltage restorers are utilized (Sahand \& Mohammad 2019: Gregory et. al., 2017; Sadigh and Smedley 2016). The three-leg topology is only here and there favored inferable from a smaller number of switching devices and lower switching losses contrasted with the fourleg and six-leg topologies. Anyway, higher-order harmonics produced in the 4-leg and 6-leg topologies because of regular exchanging of semiconductor switches can be wiped out by the utilization of RL high pass filter (Sadigh and Smedley 2016) and exchanging misfortunes happening in the IGBT switches can likewise be dispensed with by the utilization of DC-interface voltage controller (Ahmed et al., 2020). The impedance or Z-source inverter has an emerging inverter and has a capacity to work as a step-down and step-up inverter like voltage and current source inverters (Taheri et al., 2020). Since ZSI is considered as another kind of inverter, a great deal of research around there has been expanded (Tiago et. al. 2020; Ali 2018; Rawat and Vadhera 2019). Renewable Energy (RE) technologies based on Solar and Wind Energy resources is highly pertinent in the present environmental scenario of 
the world. The dependence on conventional energy resources such as coal and petroleum increases the risk of global warming and environmental challenges. The research and development on integrating RE resources into the energy mix of world countries are also active. As compared to the conventional power plants, renewable-based generation utilizes a different set of technologies for electricity generation and interconnection with the grid (Ahmed \& Abo 2020; Mahiraj \& Shelly 2019). However, these methods could not function admirably if the perturb and observe algorithm calculation is not been adjusted to get more power from the solar system and furthermore to keep up the voltage at the DC connection of the inverter input (Mohamed et. al. 2021; Alik and Jusoh 2018; Jyotirmaya et. al 2018). This paper discusses the solar photovoltaic (PV) fed different topologies such as Three-Leg (3-L), Four-Leg (4-L), and Six-Leg (6-L) ZSI-DVR. For getting a maximum output power from the solar panel under change of irradiation of the sun as well as the ambient temperature of the solar panel Perturb \& Observe (P\&O) is used. To show the performance comparison of different topologies, the topologies are simulated in a MATLAB/SIMULINK platform. The obtained simulation results are comparable but even slightly better in six-leg ZSI based DVR compared to three-leg, and four-Leg ZSI based DVRs.

\section{VOLTAGE SWELL}

Voltage Swell is defined by IEEE 1159 as the increase in the RMS voltage level to $110 \%-180 \%$ of nominal, at the power frequency for durations of $1 / 2$ cycle to one (1) minute. The voltage sag and its characteristics are highlighted in Figure 1 (Saeed \& Raheef 2021). Voltage swells are the opposite of voltage sags (dips) and they are defined as a momentary increase in RMS voltage of $10 \%$ or more above specify (rated) equipment voltage for a period of $1 / 2$ cycle to $1 \mathrm{~min}$, as defined in the IEC 61000-4-30 standard.

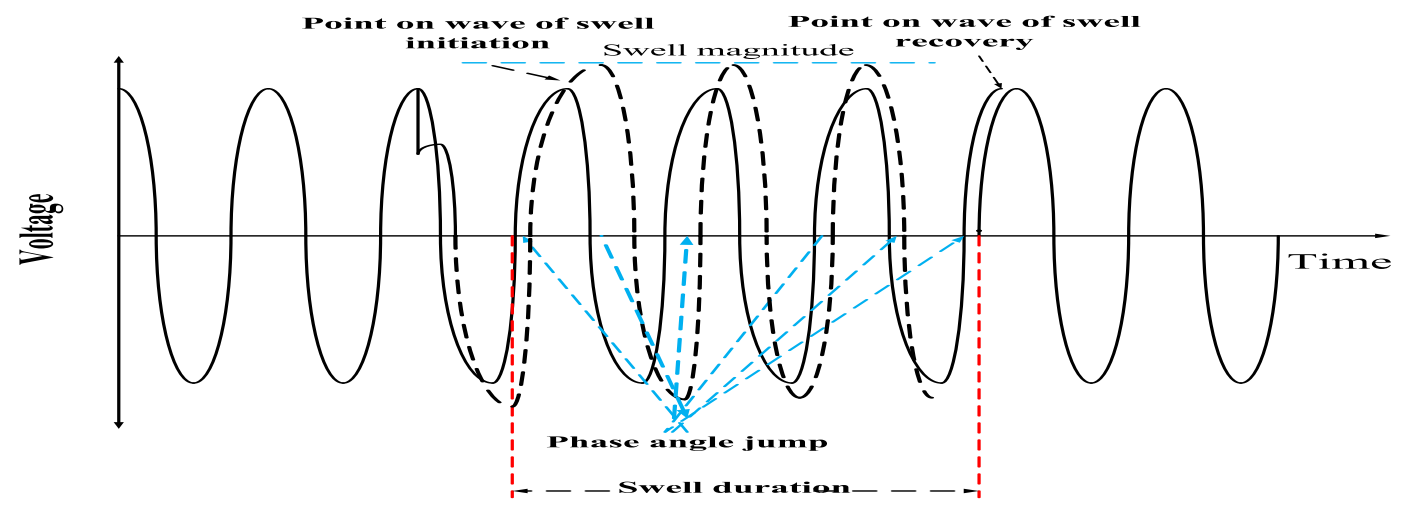

Figure 1. Voltage swell.

\section{ECONOMICAL AND TECHNICAL IMPACTS OF POWER QUALITY}

\section{Economical Impacts of Power Quality}

The economic impacts because of power quality issues are wide. This is especially the situation for sag, swell, and interruptions. The power quality can be separated into direct and indirect costs (Sharmaa et. al., 2018).

\section{Direct Economic Impacts}

i. Loss of production

ii. Unrecoverable downtime and resources 
iii. Process restart costs

iv. Spoilage of semi-finished production

v. Equipment damage

vi. Direct costs associated with human health and safety

vii. Financial penalties incurred through non-fulfilment of contracts

viii. Environmental financial penalties

ix. Utility costs associated with the interruption.

\section{Indirect Economic Impacts}

i. The costs to an organization of revenue/income being postponed

ii. The financial cost of loss of market share

iii. The cost of restoring brand equity.

\section{Technical Impacts of Power Quality}

The technical impacts because of power quality problems are wide such as unexpected power supply failures (breakers tripping, fuses blowing), equipment failure or malfunctioning, Equipment overheating (transformers, motors) leading to their lifetime reduction, damage to sensitive equipment (PCs, production line control systems), electronic communication interferences, and increase of system losses (Sharmaa et. al., 2018).

\section{DIFFERENT TOPOLOGIES OF Z-SOURCE INVERTER BASED DVRS THREE-PHASE THREE-LEG ZSI-DVR}

The three-leg ZSI based DVR configuration is shown in Figure 2. The advantage of the isolated ZSI based DVR topology is that the voltage rating of the ZSI can be optimally designed as this is an interfacing transformer. The three single-phase three-Leg ZSI based DVR requires six switches. The neutral isolation transformers $\mathrm{Tr}-1, \mathrm{Tr}-$ 2 , and $\operatorname{Tr}-3$ and the neutral three-phase supply and neutral of the load are connected for the elimination of higher neutral currents in the three-phase four-wire systems (3-leg, 4-Leg, and 6-Leg).

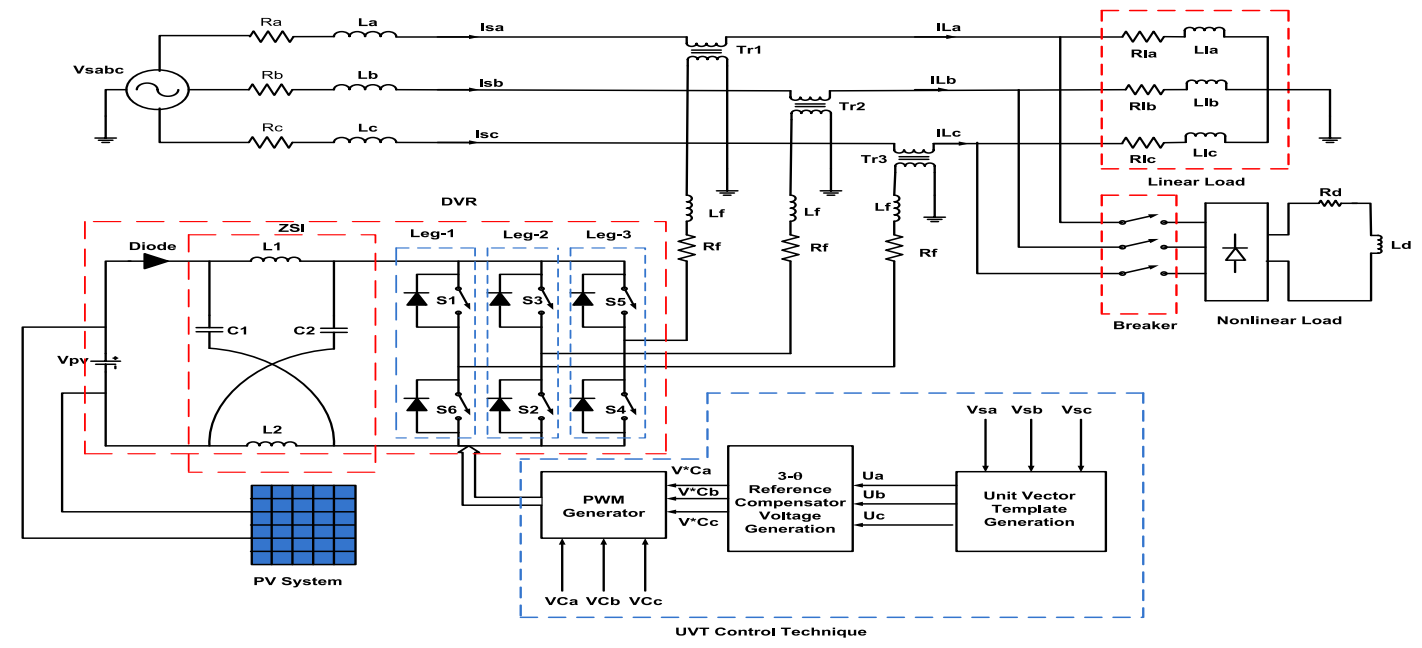

Figure 2 Three-Leg ZSI-DVR 


\section{MODULATION OF THREE-LEG ZSI-DVR}

Figure 3 shows the exchanging state sequence of a 3-leg ZSI-DVR, where three state advances happen (for example null $\{000\} \rightarrow$ active $\{100\} \rightarrow$ active $\{110\} \rightarrow$ null $\{111\})$ and the null states toward the beginning and end of an exchanging cycle ' $t$ ' length equivalent time spans. Table 1 shows the different exchanging conditions of a 3-leg Z-source inverter.

$\left\{\begin{array}{l}u_{\max }\left(S_{W} M\right)=u_{\text {max }}+u_{o f f}+\frac{t_{0}}{t} \\ u_{\max }\left(S_{W} N\right)=u_{\text {max }}+u_{o f f}+\frac{t_{0}}{3 t}\end{array}\right\}$

$\left\{\begin{array}{l}u_{m i d}\left(S_{W} M\right)=u_{m i d}+u_{o f f}+\frac{t_{0}}{3 t} \\ u_{\text {max }}\left(S_{W} N\right)=u_{\text {max }}+u_{o f f}-\frac{t_{0}}{3 t}\end{array}\right\}$

$\left\{\begin{array}{l}u_{\min }\left(S_{W} M\right)=u_{\text {min }}+u_{o f f}+\frac{t_{0}}{3 t} \\ u_{\min }\left(S_{W} N\right)=u_{\text {min }}+u_{o f f}-\frac{t_{0}}{t}\end{array}\right\}$

$\{M, N\}=\{1,4\},\{3,6\}$ or $\{5,2\}$

Table 1. Trading States of a 3-Leg ZSI.

(!SX Represents Complement of SX, where $\mathrm{X}=1,3$ or 5 )

\begin{tabular}{|c|c|c|c|c|c|c|}
\hline States (Output Voltage) & S1 & S1 & S3 & S4 & S5 & S6 \\
\hline Active $\{100\}$ (finite) & 1 & 0 & 0 & 1 & 0 & 1 \\
\hline Active $\{110\}$ (finite) & 1 & 0 & 1 & 0 & 0 & 1 \\
\hline Active $\{010\}$ (finite) & 0 & 1 & 1 & 0 & 0 & 1 \\
\hline Active $\{011\}$ (finite) & 0 & 1 & 1 & 0 & 1 & 0 \\
\hline Active $\{001\}$ (finite) & 0 & 1 & 0 & 1 & 1 & 0 \\
\hline Active $\{101\}$ (finite) & 1 & 0 & 0 & 1 & 1 & 0 \\
\hline Null $\{000\}(0 \mathrm{~V})$ & 0 & 1 & 0 & 1 & 0 & 1 \\
\hline Null $\{111\}(0 \mathrm{~V})$ & 1 & 0 & 1 & 0 & 1 & 0 \\
\hline Shoot-Through E1 (0 V) & 1 & 1 & & & & \\
\hline Shoot-Through E2 (0 V) & $\mathrm{S} 1$ & $! \mathrm{S} 1$ & 1 & 1 & $\mathrm{~S} 5$ & !S5 \\
\hline Shoot-through E3 $(0 \mathrm{~V})$ & $\mathrm{S} 1$ & !S1 & $\mathrm{S} 3$ & !S3 & 1 & 1 \\
\hline Shoot-Through E4 $(0 \mathrm{~V})$ & 1 & 1 & 1 & 1 & $\mathrm{~S} 5$ & !S5 \\
\hline Shoot-Through E5 $(0 \mathrm{~V})$ & 1 & 1 & $\mathrm{~S} 3$ & $! \mathrm{S} 3$ & 1 & 1 \\
\hline Shoot-Through E6 $(0 \mathrm{~V})$ & $\mathrm{S} 1$ & $! \mathrm{S} 1$ & 1 & 1 & 1 & 1 \\
\hline Shoot-Through E7 $(0 \mathrm{~V})$ & 1 & 1 & 1 & 1 & 1 & 1 \\
\hline
\end{tabular}




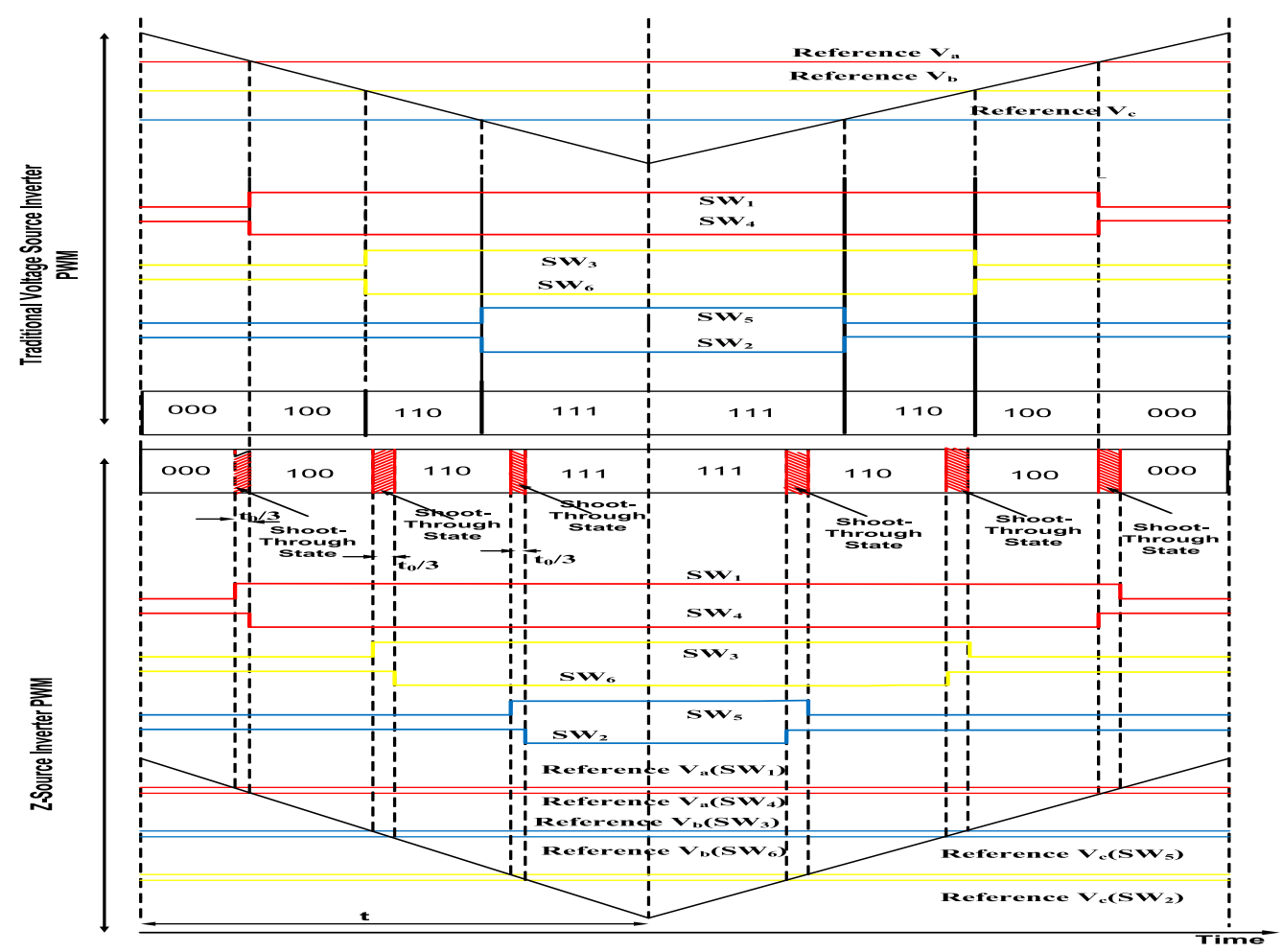

Figure 3. Modeling of Three-Leg ZSI-DVR.

\section{THREE-PHASE FOUR-LEG ZSI-DVR}

The schematic diagram of a three-phase four-leg ZSI based DVR connected to a three-phase four-wire power distribution system is shown in Fig. 4. The three single-phase four-leg ZSI DVR requires eight switches.

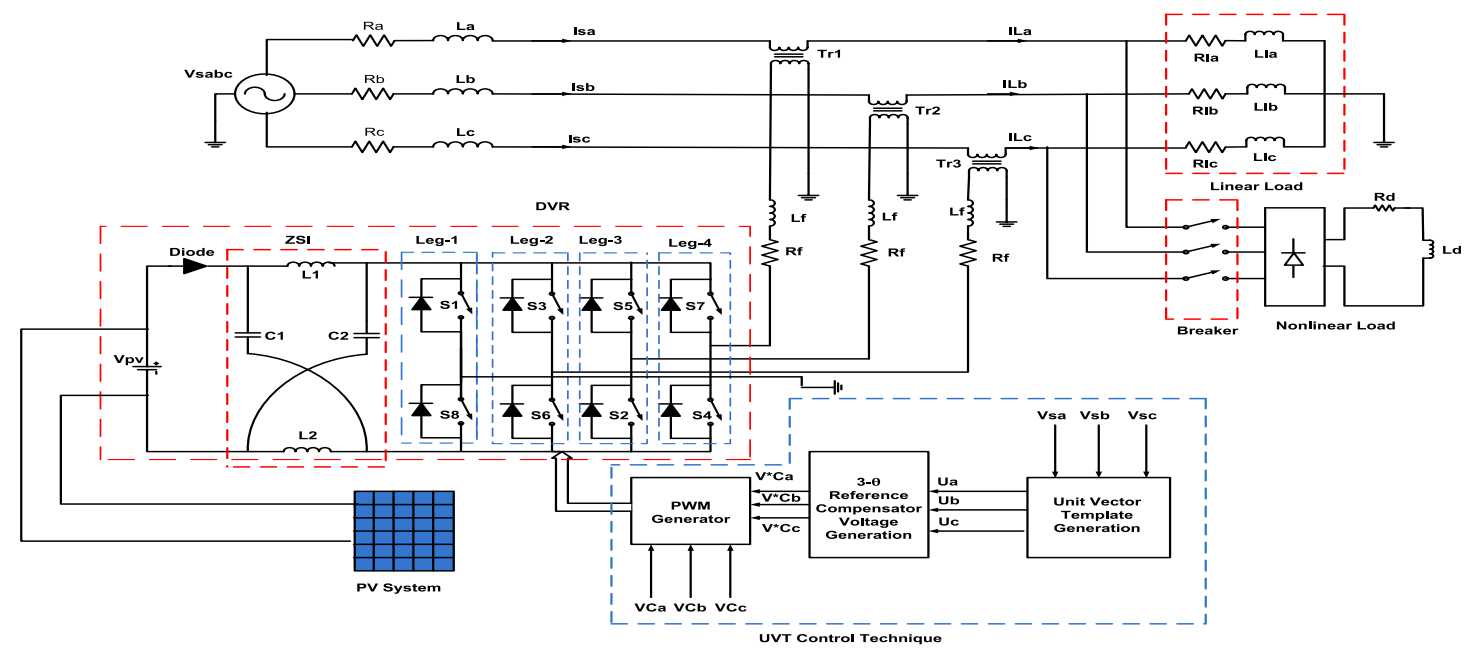

Figure 4. Four-Leg ZSI-DVR. 


\section{MODELING OF THREE-PHASE FOUR-LEG ZSI}

Figure 5 shows the exchanging conditions of a 4-leg ZSI DVR, which incorporate 14 active states, 2 null states, and 15 ST states. Among the $15 \mathrm{SW}$ states, just states F1 to F4 can be embedded at the four state changes of a fourleg Z-source inverter. In Fig 5, active state $\{1100\}$ stays fixed with states F2 and F4 of span to/4 embedded to one side and right separately during the falling transporter edge. Active states $\{1000\}$ and $\{1101\}$ are then separately leftand right-moved by t0/4 with their intervals of time kept steady. Ultimately, states F1 and F3 are added to one side of state $\{1000\}$ and right of state $\{1101\}$ individually. Table 2 shows the different exchanging conditions of a threestage four-leg impedance source inverter.

$\left\{\begin{array}{l}u_{\max }\left(S_{W} M\right)=u_{\text {max }}+u_{o f f}+t_{0} / t \\ u_{\text {max }}\left(S_{W} N\right)=u_{\text {max }}+u_{o f f}+t_{0} / 2 t\end{array}\right\}$

$\left\{\begin{array}{c}u_{m i d-1}\left(S_{W} M\right)=u_{m i d-1}+u_{o f f}+t_{0} / 2 t \\ u_{m i d-1}\left(S_{W} N\right)=u_{m i d-1}+u_{o f f}\end{array}\right\}$

$\left\{\begin{array}{c}u_{m i d-2}\left(S_{W} M\right)=u_{m i d-2}+u_{o f f} \\ u_{m i d-2}\left(S_{W} N\right)=u_{m i d-2}+u_{o f f}-\frac{t_{0}}{2 t}\end{array}\right\}$

$\left\{\begin{array}{l}u_{\min }\left(S_{W} M\right)=u_{\min }+u_{o f f}-\frac{t_{0}}{2 t} \\ u_{\min }\left(S_{W} N\right)=u_{\min }+u_{o f f}-\frac{t_{0}}{t}\end{array}\right\}$

$\{M, N\}=\{1,4\},\{3,6\},\{5,2\}$ or $\{7,8\}$

Table 2. Trading States of a 4-Leg ZSI

(!SX Represents Complement of SX, where $\mathrm{X}=1,3,5$ or 7 ).

\begin{tabular}{|c|c|c|c|c|c|c|c|c|}
\hline States (Output Voltage) & S1 & S4 & S3 & S6 & S5 & S2 & S7 & S8 \\
\hline Active $\{1000\}$ (finite) & 1 & 0 & 0 & 1 & 0 & 1 & 0 & 1 \\
\hline Active $\{1001\}$ (finite) & 1 & 0 & 0 & 1 & 0 & 1 & 1 & 0 \\
\hline Active $\{1100\}$ (finite) & 1 & 0 & 1 & 0 & 0 & 1 & 0 & 1 \\
\hline Active $\{1101\}$ (finite) & 1 & 0 & 1 & 0 & 0 & 1 & 1 & 0 \\
\hline Active $\{0100\}$ (finite) & 0 & 1 & 1 & 0 & 0 & 1 & 0 & 1 \\
\hline Active $\{0101\}$ (finite) & 0 & 1 & 1 & 0 & 0 & 1 & 1 & 0 \\
\hline Active $\{0110\}$ (finite) & 0 & 1 & 1 & 0 & 1 & 0 & 0 & 1 \\
\hline Active $\{0111\}$ (finite) & 0 & 1 & 1 & 0 & 1 & 0 & 1 & 0 \\
\hline Active $\{0010\}$ (finite) & 0 & 1 & 0 & 1 & 1 & 0 & 0 & 1 \\
\hline Active $\{0011\}$ (finite) & 0 & 1 & 0 & 1 & 1 & 0 & 1 & 0 \\
\hline
\end{tabular}




\begin{tabular}{|c|c|c|c|c|c|c|c|c|}
\hline Active $\{1010\}$ (finite) & 1 & 0 & 0 & 1 & 1 & 0 & 0 & 1 \\
\hline Active $\{1011\}$ (finite) & 1 & 0 & 0 & 1 & 1 & 0 & 1 & 0 \\
\hline Active $\{0001\}$ (finite) & 0 & 1 & 0 & 1 & 0 & 1 & 1 & 0 \\
\hline Active $\{1110\}$ (finite) & 1 & 0 & 1 & 0 & 1 & 0 & 0 & 1 \\
\hline Null $\{000\}(0 \mathrm{~V})$ & 0 & 1 & 0 & 1 & 0 & 1 & 0 & 1 \\
\hline Null $\{111\}(0 \mathrm{~V})$ & 1 & 0 & 1 & 0 & 1 & 0 & 1 & 0 \\
\hline Shoot-Through F1 $(0 \mathrm{~V})$ & 1 & 1 & $\mathrm{~S} 3$ & !S3 & $\mathrm{S} 5$ & !S5 & $\mathrm{S} 7$ & !S7 \\
\hline Shoot-Through F2 $(0 \mathrm{~V})$ & $\mathrm{S} 1$ & !S1 & 1 & 1 & $\mathrm{~S} 5$ & !S5 & $\mathrm{S} 7$ & !S7 \\
\hline Shoot-through F3 $(0 \mathrm{~V})$ & $\mathrm{S} 1$ & !S1 & $\mathrm{S} 3$ & !S3 & 1 & 1 & $\mathrm{~S} 7$ & !S7 \\
\hline Shoot-Through F4 $(0 \mathrm{~V})$ & $\mathrm{S} 1$ & !S1 & $\mathrm{S} 3$ & !S3 & $\mathrm{S} 5$ & !S5 & 1 & 1 \\
\hline Shoot-Through F5 $(0 \mathrm{~V})$ & 1 & 1 & 1 & 1 & $\mathrm{~S} 5$ & !S5 & $\mathrm{S} 7$ & !S7 \\
\hline Shoot-Through F6 $(0 \mathrm{~V})$ & 1 & 1 & $\mathrm{~S} 3$ & !S3 & 1 & 1 & $\mathrm{~S} 7$ & !S7 \\
\hline Shoot-Through F7 $(0 \mathrm{~V})$ & 1 & 1 & $\mathrm{~S} 3$ & !S3 & $\mathrm{S} 5$ & !S5 & 1 & 1 \\
\hline Shoot-Through F8 $(0 \mathrm{~V})$ & $\mathrm{S} 1$ & !S1 & 1 & 1 & 1 & 1 & $\mathrm{~S} 7$ & !S7 \\
\hline Shoot-Through F9 $(0 \mathrm{~V})$ & $\mathrm{S} 1$ & !S1 & 1 & 1 & $\mathrm{~S} 5$ & !S5 & 1 & 1 \\
\hline Shoot-Through F10 $(0 \mathrm{~V})$ & $\mathrm{S} 1$ & !S1 & $\mathrm{S} 3$ & !S3 & 1 & 1 & 1 & 1 \\
\hline Shoot-Through F11 $(0 \mathrm{~V})$ & 1 & 1 & 1 & 1 & 1 & 1 & $\mathrm{~S} 7$ & !S7 \\
\hline Shoot-Through F12 $(0 \mathrm{~V})$ & 1 & 1 & 1 & 1 & $\mathrm{~S} 5$ & !S5 & 1 & 1 \\
\hline Shoot-Through F13 $(0 \mathrm{~V})$ & 1 & 1 & & & 1 & 1 & 1 & 1 \\
\hline Shoot-Through F14 $(0 \mathrm{~V})$ & $\mathrm{S} 1$ & !S1 & 1 & 1 & 1 & 1 & 1 & 1 \\
\hline Shoot-Through F15 $(0 \mathrm{~V})$ & 1 & 1 & 1 & 1 & 1 & 1 & 1 & 1 \\
\hline
\end{tabular}

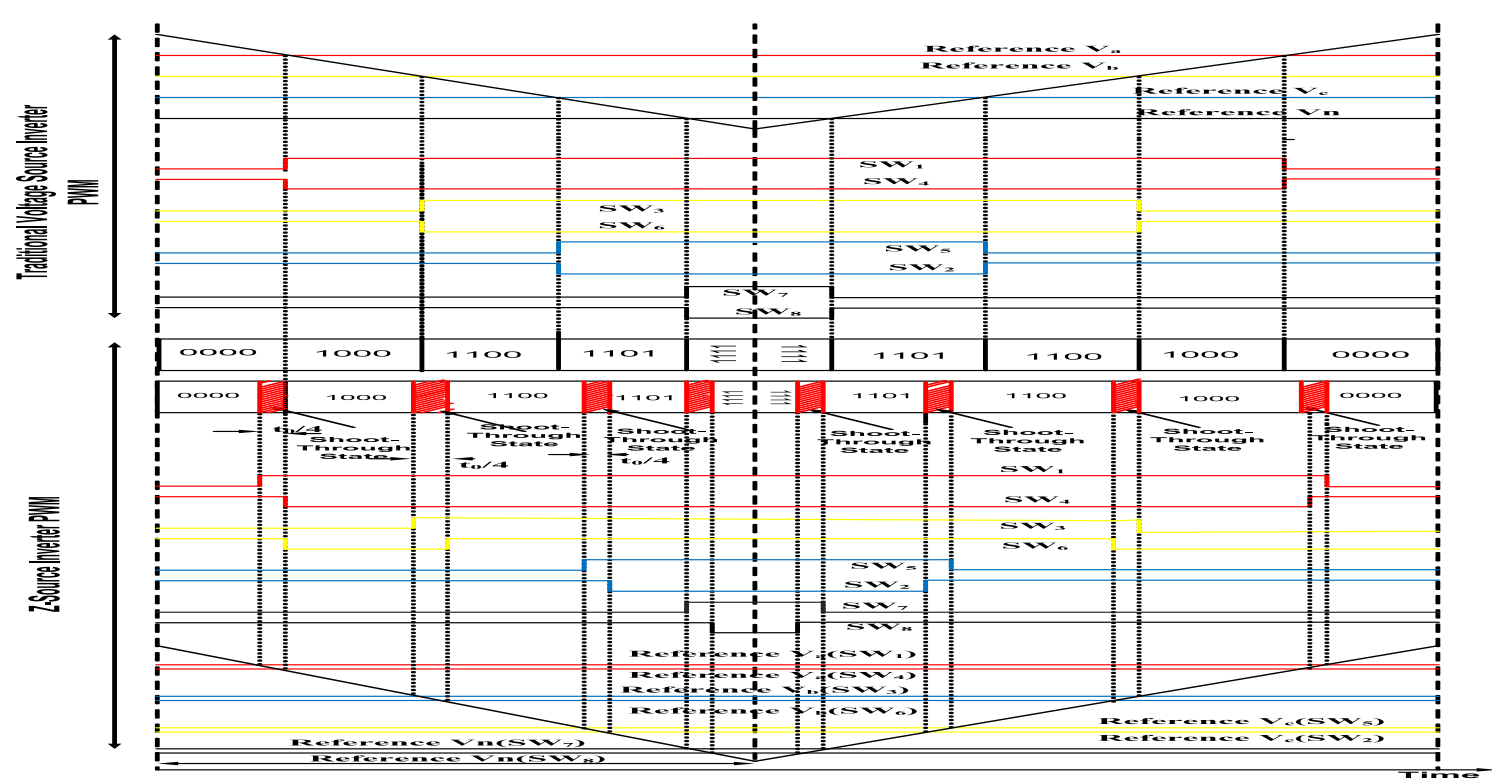

Figure 5. Modeling of Four-Leg ZSI-DVR. 


\section{THREE-PHASE SIX-LEG ZSI DVR}

The schematic diagram of a three-phase six-leg ZSI based DVR connected to a three-phase four-wire power distribution system is shown in Fig. 6. The three-single phase six-leg ZSI DVR requires twelve switches. Table 3 shows the various switching states of a three-phase six-leg impedance source inverter.

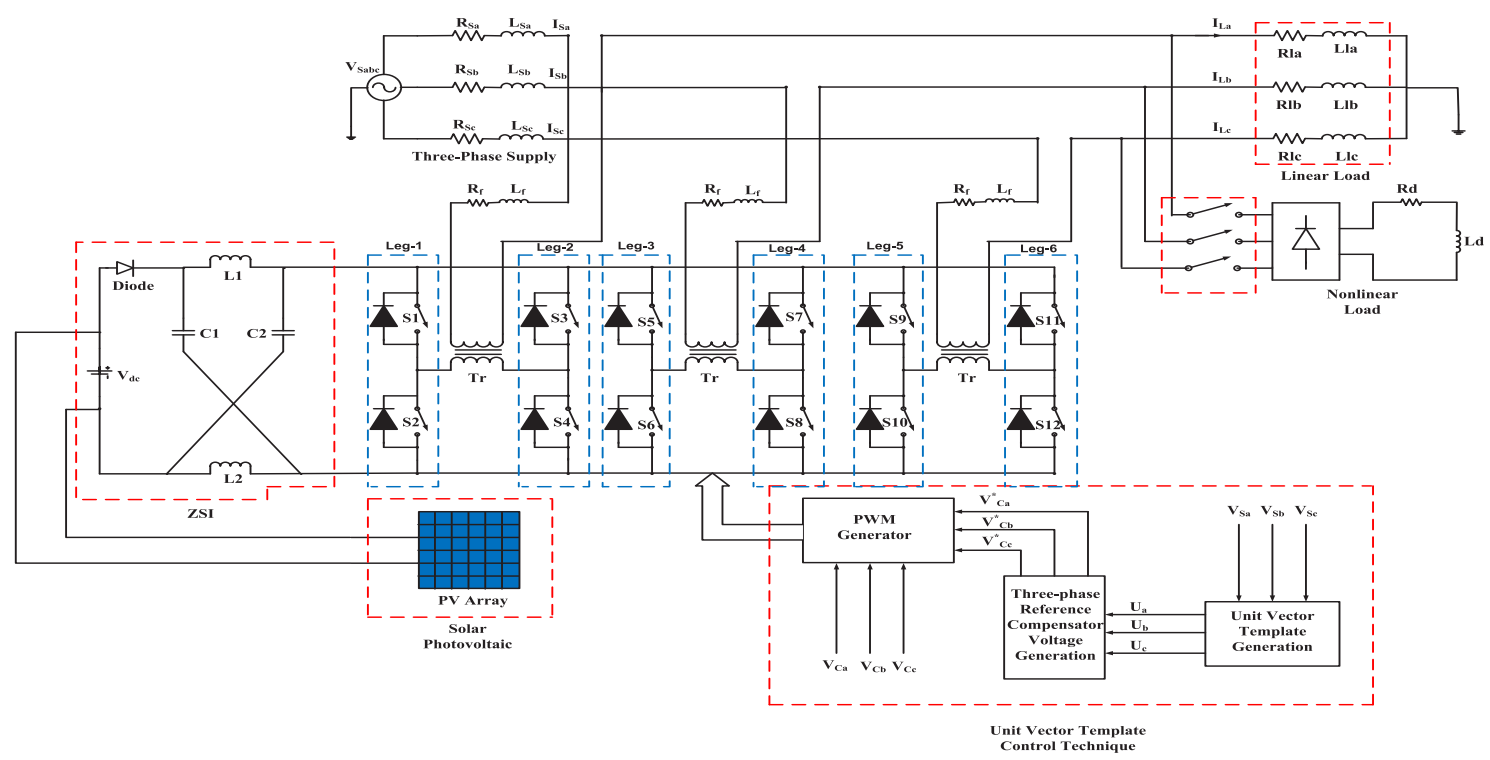

Figure 6. Six-leg ZSI-DVR.

\section{MODELING OF THREE-PHASE FOUR-LEG ZSI}

Figure 7 shows the construction of the 83 exchanging conditions of a proposed Z-Source inverter-based DVR, which incorporates forty active states, two null states, and 41 shoot-through states. The active and null states wherein the two switches of a stage leg are exchanged integral and are normal to both traditional inverters and Z-source inverters. Be that as it may, the excess 41 shoot-through states where one (shoot-through states F1 and F40) or a few or four or five or six-stage legs (shoot-through state F41) are short-circuited are one of a kind to the proposed Zsource inverter. Table 3 shows the different exchanging conditions of a three-stage 6-leg impedance source inverter.

$\left\{\begin{array}{l}u_{\max }\left(S_{W} M\right)=u_{\max }+u_{o f f}+t_{0} / t \\ u_{\max }\left(S_{W} N\right)=u_{\max }+u_{o f f}+t_{0} / 2 t\end{array}\right\}$

$\left\{\begin{array}{c}u_{m i d-1}\left(S_{W} M\right)=u_{m i d-1}+u_{o f f}+t_{0} / 2 t \\ u_{m i d-1}\left(S_{W} N\right)=u_{m i d-1}+u_{o f f}\end{array}\right\}$

$\left\{\begin{array}{c}u_{m i d-2}\left(S_{W} M\right)=u_{m i d-2}+u_{o f f} \\ u_{m i d-2}\left(S_{W} N\right)=u_{m i d-2}+u_{o f f}-t_{0} / 2 t\end{array}\right\}$ 


$$
\begin{gathered}
\left\{\begin{array}{l}
u_{\text {mid }-3}\left(S_{W} M\right)=u_{\text {mid-3 }}+u_{o f f}-t_{0} / 2 t \\
u_{\text {mid-3 }}\left(S_{W} N\right)=u_{\text {mid-3 }}+u_{o f f}-t_{0} / t
\end{array}\right\} \\
\left\{\begin{array}{l}
u_{\text {mid-4 }}\left(S_{W} M\right)=u_{\text {mid-4 }}+u_{o f f}-t_{0} / t \\
u_{\text {mid-4 }}\left(S_{W} N\right)=u_{\text {mid-4 }}+u_{o f f}-t_{0} / 2 t
\end{array}\right\} \\
\left\{\begin{array}{l}
u_{\min }\left(S_{W} M\right)=u_{\text {min }}+u_{o f f}-t_{0} / 2 t \\
u_{\min }\left(S_{W} N\right)=u_{\text {min }}+u_{o f f}-t_{0} / t
\end{array}\right\}
\end{gathered}
$$

$\{M, N\}=\{1,4\},\{3,6\},\{5,2\},\{7,8\},\{9,10\}$ or $\{11,12\}$

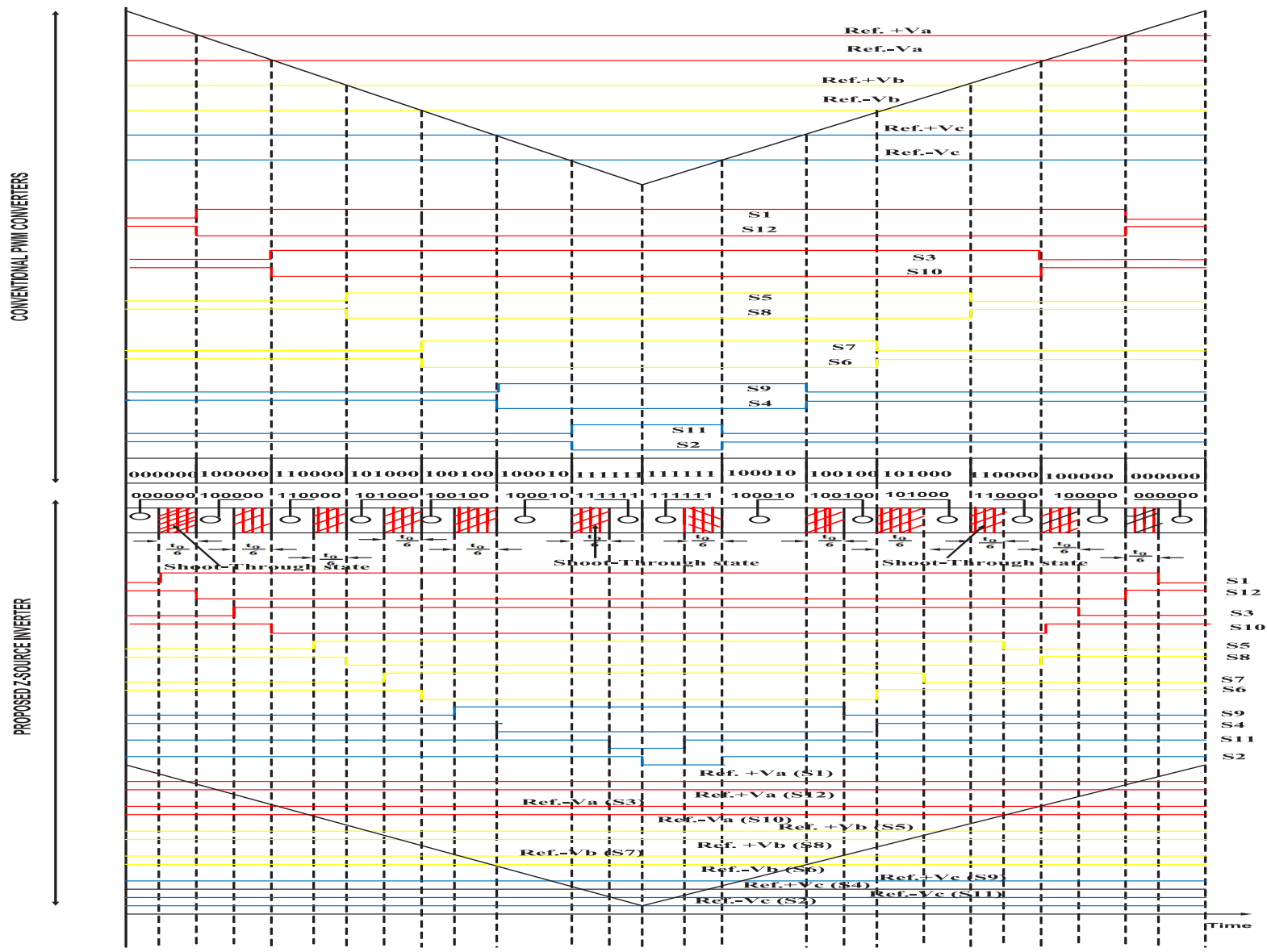

Figure 7. Modulation of the Proposed Z-Source Inverter. 
Table 3. Trading States of proposed 6-Leg ZSI.

(!SX represents complement of SX, whrer $\mathrm{X}=1,3,5,7,9$ OR 11)

\begin{tabular}{|c|c|c|c|c|c|c|c|c|c|c|c|c|}
\hline State $\{100000\}$ (finite) & S1 & $\mathrm{S} 12$ & S3 & S10 & S5 & S8 & S7 & S6 & S9 & S4 & S11 & S2 \\
\hline State $\{110000\}$ (finite) & 1 & 0 & 0 & 1 & 0 & 1 & 0 & 1 & 0 & 1 & 0 & 1 \\
\hline State $\{101000\}$ (finite) & 1 & 0 & 1 & 0 & 0 & 1 & 0 & 1 & 0 & 1 & 0 & 1 \\
\hline State $\{100100\}$ (finite) & 1 & 0 & 0 & 1 & 1 & 0 & 0 & 1 & 0 & 1 & 0 & 1 \\
\hline State $\{100010\}$ (finite) & 1 & 0 & 0 & 1 & 0 & 1 & 0 & 1 & 1 & 0 & 0 & 1 \\
\hline State $\{100001\}$ (finite) & 1 & 0 & 0 & 1 & 0 & 1 & 0 & 1 & 0 & 1 & 1 & 0 \\
\hline State $\{010000\}$ finite & 0 & 1 & 1 & 0 & 0 & 1 & 0 & 1 & 0 & 1 & 0 & 1 \\
\hline State $\{011000\}$ finite & 0 & 1 & 1 & 0 & 1 & 0 & 0 & 1 & 0 & 1 & 0 & 1 \\
\hline State $\{010100\}$ finite & 0 & 1 & 1 & 0 & 0 & 1 & 1 & 0 & 0 & 1 & 0 & 1 \\
\hline State $\{010010\}$ finite & 0 & 1 & 1 & 0 & 0 & 1 & 0 & 1 & 1 & 0 & 0 & 1 \\
\hline State $\{010001\}$ finite & 0 & 1 & 1 & 0 & 0 & 1 & 0 & 1 & 0 & 1 & 1 & 0 \\
\hline State $\{001000\}$ finite & 0 & 1 & 0 & 1 & 1 & 0 & 0 & 1 & 0 & 1 & 0 & 1 \\
\hline State $\{001100\}$ finite & 0 & 1 & 0 & 1 & 1 & 0 & 1 & 0 & 0 & 1 & 0 & 1 \\
\hline State $\{001010\}$ finite & 0 & 1 & 0 & 1 & 1 & 0 & 0 & 1 & 1 & 0 & 0 & 1 \\
\hline State $\{001001\}$ finite & 0 & 1 & 0 & 1 & 1 & 0 & 0 & 1 & 0 & 1 & 1 & 0 \\
\hline State $\{000100\}$ finite & 0 & 1 & 0 & 1 & 0 & 1 & 1 & 0 & 0 & 1 & 0 & 1 \\
\hline State $\{000110\}$ finite & 0 & 1 & 0 & 1 & 0 & 1 & 1 & 0 & 1 & 0 & 0 & 1 \\
\hline State $\{000101\}$ finite & 0 & 1 & 0 & 1 & 0 & 1 & 1 & 0 & 0 & 1 & 1 & 0 \\
\hline State $\{000010\}$ finite & 0 & 1 & 0 & 1 & 0 & 1 & 0 & 1 & 1 & 0 & 0 & 1 \\
\hline State $\{000011\}$ finite & 0 & 1 & 0 & 1 & 0 & 1 & 0 & 1 & 1 & 0 & 1 & 0 \\
\hline State $\{111000\}$ finite & 1 & 0 & 1 & 0 & 1 & 0 & 0 & 1 & 0 & 1 & 0 & 1 \\
\hline State $\{110100\}$ finite & 1 & 0 & 1 & 0 & 0 & 1 & 1 & 0 & 0 & 1 & 0 & 1 \\
\hline State $\{110010\}$ finite & 1 & 0 & 1 & 0 & 0 & 1 & 0 & 1 & 1 & 0 & 0 & 1 \\
\hline State $\{110001\}$ finite & 1 & 0 & 1 & 0 & 0 & 1 & 0 & 1 & 0 & 1 & 1 & 0 \\
\hline State $\{011100\}$ finite & 0 & 1 & 1 & 0 & 1 & 0 & 1 & 0 & 0 & 1 & 0 & 1 \\
\hline State $\{011010\}$ finite & 0 & 1 & 1 & 0 & 1 & 0 & 0 & 1 & 1 & 0 & 0 & 1 \\
\hline State $\{011001\}$ finite & 0 & 1 & 1 & 0 & 1 & 0 & 0 & 1 & 0 & 1 & 1 & 0 \\
\hline State $\{001110\}$ finite & 0 & 1 & 0 & 1 & 1 & 0 & 1 & 0 & 1 & 0 & 0 & 1 \\
\hline State $\{001101\}$ finite & 0 & 1 & 0 & 1 & 1 & 0 & 1 & 0 & 0 & 1 & 1 & 0 \\
\hline State $\{000111\}$ finite & 0 & 1 & 0 & 1 & 0 & 1 & 1 & 0 & 1 & 0 & 1 & 0 \\
\hline State $\{100011\}$ finite & 1 & 0 & 0 & 1 & 0 & 1 & 0 & 1 & 1 & 0 & 1 & 0 \\
\hline State $\{111100\}$ finite & 1 & 0 & 1 & 0 & 1 & 0 & 1 & 0 & 0 & 1 & 0 & 1 \\
\hline State $\{111010\}$ finite & 1 & 0 & 1 & 0 & 1 & 0 & 0 & 1 & 1 & 0 & 0 & 1 \\
\hline State $\{111001\}$ finite & 1 & 0 & 1 & 0 & 1 & 0 & 0 & 1 & 0 & 1 & 1 & 0 \\
\hline
\end{tabular}




\begin{tabular}{|c|c|c|c|c|c|c|c|c|c|c|c|c|}
\hline State $\{011110\}$ finite & 0 & 1 & 1 & 0 & 1 & 0 & 1 & 0 & 1 & 0 & 0 & 1 \\
\hline State $\{011101\}$ finite & 0 & 1 & 1 & 0 & 1 & 0 & 1 & 1 & 0 & 1 & 1 & 0 \\
\hline State $\{001111\}$ finite & 0 & 1 & 0 & 1 & 1 & 0 & 1 & 0 & 1 & 0 & 1 & 0 \\
\hline State $\{100111\}$ finite & 1 & 0 & 0 & 1 & 0 & 1 & 1 & 0 & 1 & 0 & 1 & 0 \\
\hline State $\{111110\}$ finite & 1 & 0 & 1 & 0 & 1 & 0 & 1 & 0 & 1 & 0 & 0 & 1 \\
\hline State $\{011111\}$ finite & 0 & 1 & 1 & 0 & 1 & 0 & 1 & 0 & 1 & 0 & 1 & 0 \\
\hline Null $\{000000\}(0 \mathrm{~V})$ & 0 & 1 & 0 & 1 & 0 & 1 & 0 & 1 & 0 & 1 & 0 & 1 \\
\hline Null $\{111111\}(0 \mathrm{~V})$ & 1 & 0 & 1 & 0 & 1 & 0 & 1 & 0 & 1 & 0 & 1 & 0 \\
\hline Shoot-through F1 (0V) & 1 & 1 & $\mathrm{~S} 3$ & !S3 & S5 & !S5 & S7 & !S7 & S9 & !S9 & S11 & !S11 \\
\hline Shoot-through F2 (0V) & $\mathrm{S} 1$ & !S1 & 1 & 1 & S5 & !S5 & S7 & !S7 & S9 & !S9 & S11 & !S11 \\
\hline Shoot-through F3 (0V) & S1 & !S1 & $\mathrm{S} 3$ & !S3 & 1 & 1 & S7 & !S7 & S9 & !S9 & S11 & !S11 \\
\hline Shoot-through F4 (0V) & $\mathrm{S} 1$ & !S1 & $\mathrm{S} 3$ & !S3 & S5 & !S5 & 1 & 1 & S9 & !S9 & S11 & !S11 \\
\hline Shoot-through F5 (0V) & S1 & !S1 & S3 & !S3 & S5 & !S5 & S7 & !S7 & 1 & 1 & S11 & $! \mathrm{S} 11$ \\
\hline Shoot-through F6 (0V) & S1 & !S1 & S3 & !S3 & S5 & !S5 & S7 & !S7 & S9 & !S9 & 1 & 1 \\
\hline Shoot-through F7 (0V) & 1 & 1 & 1 & 1 & S5 & !S5 & S7 & !S7 & S9 & !S9 & S11 & !S11 \\
\hline Shoot-through F8 (0V) & 1 & 1 & $\mathrm{~S} 3$ & !S3 & 1 & 1 & S7 & !S7 & S9 & !S9 & S11 & !S11 \\
\hline Shoot-through F9 (0V) & 1 & 1 & S3 & !S3 & S5 & !S5 & 1 & 1 & S9 & !S9 & S11 & !S11 \\
\hline Shoot-through F10(0V) & 1 & 1 & $\mathrm{~S} 3$ & !S3 & S5 & !S5 & S7 & !S7 & 1 & 1 & S11 & !S11 \\
\hline Shoot-through F11(0V) & 1 & 1 & $\mathrm{~S} 3$ & !S3 & S5 & !S5 & S7 & !S7 & S9 & !S9 & 1 & 1 \\
\hline Shoot-through F12(0V) & $\mathrm{S} 1$ & !S1 & 1 & 1 & 1 & 1 & S7 & !S7 & S9 & !S9 & S11 & !S11 \\
\hline Shoot-through F13(0V) & $\mathrm{S} 1$ & !S1 & 1 & 1 & S5 & !S5 & 1 & 1 & S9 & !S9 & S11 & !S11 \\
\hline Shoot-throughF14 (0V) & S1 & !S1 & 1 & 1 & S5 & !S5 & S7 & !S7 & 1 & 1 & S11 & !S11 \\
\hline Shoot-through F15(0V) & $\mathrm{S} 1$ & !S1 & 1 & 1 & S5 & !S5 & S7 & !S7 & S9 & !S9 & 1 & 1 \\
\hline Shoot-through F16(0V) & $\mathrm{S} 1$ & !S1 & $\mathrm{S} 3$ & !S3 & 1 & 1 & 1 & 1 & S9 & !S9 & S11 & !S11 \\
\hline Shoot-through F17(0V) & $\mathrm{S} 1$ & !S1 & $\mathrm{S} 3$ & !S3 & 1 & 1 & S7 & !S7 & 1 & 1 & S11 & !S11 \\
\hline Shoot-through F18(0V) & $\mathrm{S} 1$ & !S1 & S3 & !S3 & 1 & 1 & S7 & !S7 & S9 & !S9 & 1 & 1 \\
\hline Shoot-through F19(0V) & $\mathrm{S} 1$ & !S1 & S3 & !S3 & S5 & !S5 & 1 & 1 & 1 & 1 & S11 & !S11 \\
\hline Shoot-through F20(0V) & $\mathrm{S} 1$ & !S1 & $\mathrm{S} 3$ & !S3 & S5 & !S5 & 1 & 1 & S9 & !S9 & 1 & 1 \\
\hline Shoot-through F21(0V) & $\mathrm{S} 1$ & !S1 & S3 & !S3 & S5 & !S5 & S7 & !S7 & 1 & 1 & 1 & 1 \\
\hline Shoot-through F22(0V) & 1 & 1 & 1 & 1 & 1 & 1 & S7 & !S7 & S9 & !S9 & S11 & !S11 \\
\hline Shoot-through F23(0V) & 1 & 1 & 1 & 1 & S5 & !S5 & 1 & 1 & S9 & !S9 & S11 & !S11 \\
\hline Shoot-through F24(0V) & 1 & 1 & 1 & 1 & S5 & !S5 & S7 & !S7 & 1 & 1 & S11 & !S11 \\
\hline Shoot-through F25(0V) & 1 & 1 & 1 & 1 & S5 & !S5 & S7 & !S7 & S9 & !S9 & 1 & 1 \\
\hline Shoot-through F26(0V) & S1 & $! \mathrm{S} 1$ & 1 & 1 & 1 & 1 & 1 & 1 & S9 & !S9 & S11 & !S11 \\
\hline Shoot-through F27(0V) & $\mathrm{S} 1$ & !S1 & 1 & 1 & 1 & 1 & S7 & !S7 & 1 & 1 & S11 & !S11 \\
\hline Shoot-through F28(0V) & $\mathrm{S} 1$ & !S1 & 1 & 1 & 1 & 1 & S7 & !S7 & S9 & !S9 & 1 & 1 \\
\hline
\end{tabular}




\begin{tabular}{|l|c|c|c|c|c|c|c|c|c|c|c|c|}
\hline Shoot-through F29(0V) & $\mathrm{S} 1$ & !S1 & $\mathrm{S} 3$ & !S3 & 1 & 1 & 1 & 1 & 1 & 1 & $\mathrm{~S} 11$ & !S11 \\
\hline Shoot-through F30(0V) & $\mathrm{S} 1$ & !S1 & $\mathrm{S} 3$ & !S3 & 1 & 1 & 1 & 1 & $\mathrm{~S} 9$ & !S9 & 1 & 1 \\
\hline Shoot-through F31(0V) & $\mathrm{S} 1$ & !S1 & $\mathrm{S} 3$ & !S3 & $\mathrm{S} 5$ & !S5 & 1 & 1 & 1 & 1 & 1 & 1 \\
\hline Shoot-through F32(0V) & 1 & 1 & 1 & 1 & 1 & 1 & 1 & 1 & $\mathrm{~S} 9$ & !S9 & $\mathrm{S} 11$ & !S11 \\
\hline Shoot-through F33(0V) & 1 & 1 & 1 & 1 & 1 & 1 & $\mathrm{~S} 7$ & !S7 & 1 & 1 & $\mathrm{~S} 11$ & !S11 \\
\hline Shoot-through F34(0V) & 1 & 1 & 1 & 1 & 1 & 1 & $\mathrm{~S} 7$ & !S7 & $\mathrm{S} 9$ & !S9 & 1 & 1 \\
\hline Shoot-through F35(0V) & $\mathrm{S} 1$ & $! \mathrm{S} 1$ & 1 & 1 & 1 & 1 & 1 & 1 & 1 & 1 & $\mathrm{~S} 11$ & !S11 \\
\hline Shoot-through F36(0V) & $\mathrm{S} 1$ & !S1 & 1 & 1 & 1 & 1 & 1 & 1 & $\mathrm{~S} 9$ & !S9 & 1 & 1 \\
\hline Shoot-through F37(0V) & $\mathrm{S} 1$ & !S1 & $\mathrm{S} 3$ & !S3 & 1 & 1 & 1 & 1 & 1 & 1 & 1 & 1 \\
\hline Shoot-through F38(0V) & 1 & 1 & 1 & 1 & 1 & 1 & 1 & 1 & 1 & 1 & $\mathrm{~S} 11$ & !S11 \\
\hline Shoot-through F39(0V) & 1 & 1 & 1 & 1 & 1 & 1 & 1 & 1 & $\mathrm{~S} 9$ & !S9 & 1 & 1 \\
\hline Shoot-through F40(0V) & $\mathrm{S} 1$ & $! \mathrm{S} 1$ & 1 & 1 & 1 & 1 & 1 & 1 & 1 & 1 & 1 & 1 \\
\hline Shoot-through F41(0V) & 1 & 1 & 1 & 1 & 1 & 1 & 1 & 1 & 1 & 1 & 1 & 1 \\
\hline
\end{tabular}

\section{PERTURB \& OBSERVE TECHNIQUE}

The P\&O strategy is normally embraced for chasing down the MPPT considering reality that it is straight forward and requires only assessment of the voltage (Vpv) and current (Ipv) of the PV framework. P\&O works by perturbing (expanding or diminishing) the deliberate photovoltaic voltage (Vpv) and contrasting the instantaneous power beforehand, then, at that point after the perturbation (Mohamed et. al., 2021; Alik \& Jusoh 2018; Jyotirmaya et. al., 2018). The algorithm of the P\&O method is depicted in Figure 8.

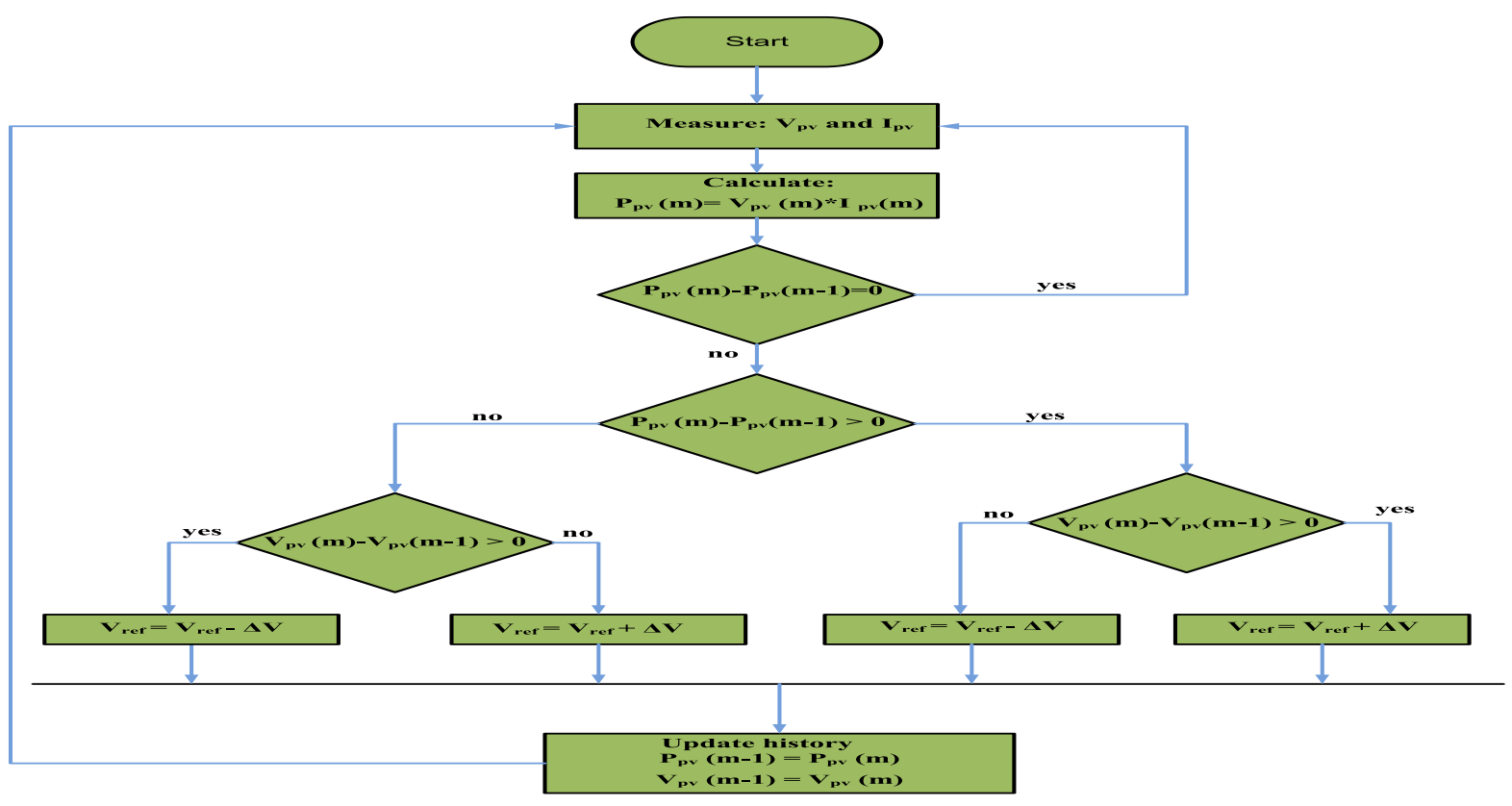

Figure 8. Stream outline of the P\&O. 


\section{RESULT AND DISCUSSION}

The proposed topologies of the DVR framework in Figures 2, 4, and 6 were set up in MATLAB/SIMULINK stage to show its presentation under unexpected exchanging a reasonable three-phase nonlinear load. Figure 9(a-c) shows the different characteristics of the solar cell under different irradiations of the sun. As the irradiation increases the open circuit voltage as well as the short circuit current also increases as shown in figures $9 \mathrm{a}$ and $\mathrm{b}$. Consequently, perturb and observe algorithm is used to get the most extreme power from the solar cell as highlighted in Figure 10.
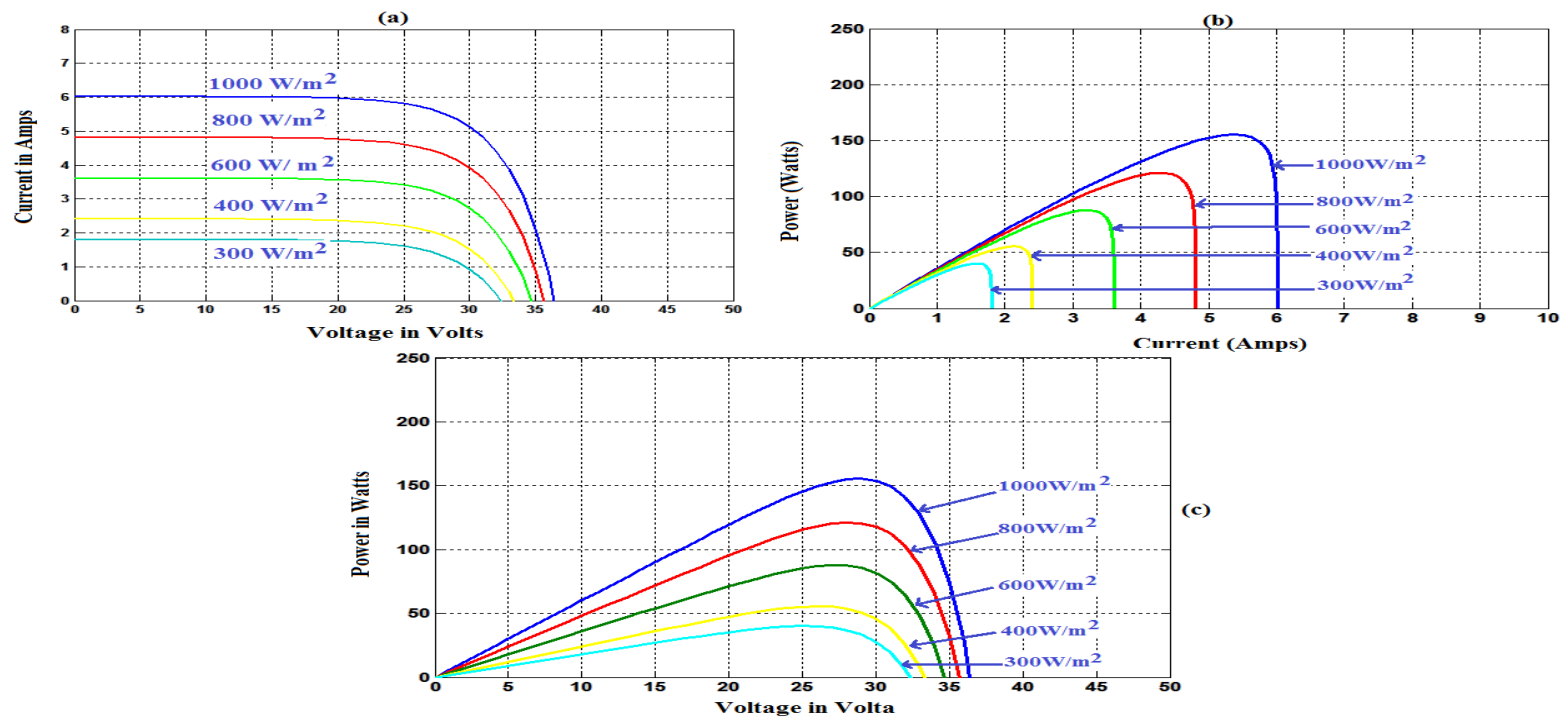

Figure 9. Characteristics of Solar Photovoltaic under various Irradiations (a) V-I characteristics (b) P-I charactristics and (c) P-V characteristics.

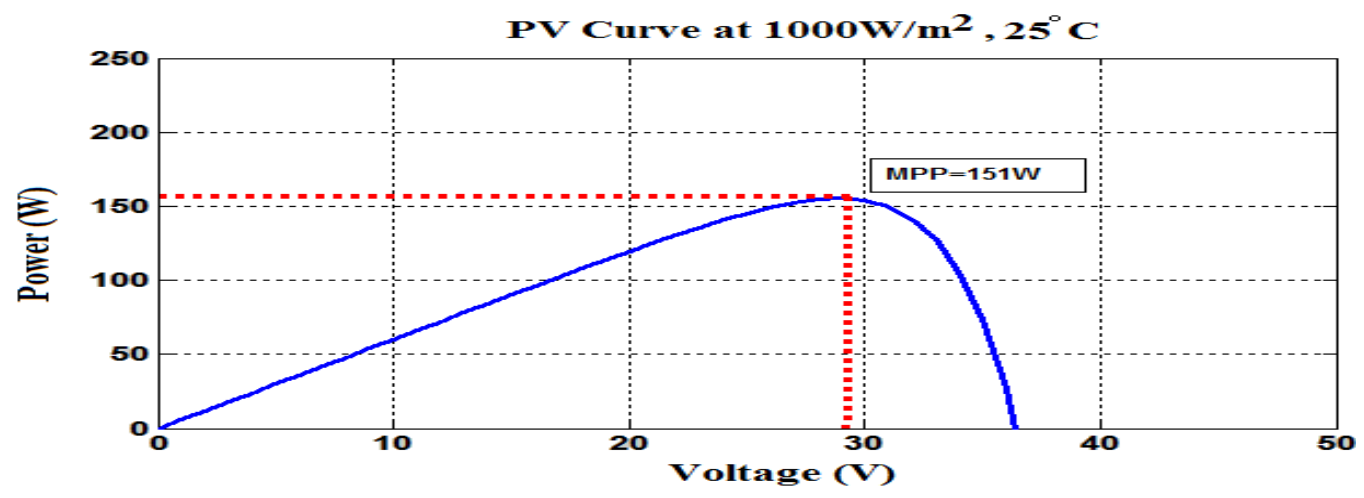

Figure 10. Maximum Power Point Tracking by Perturb \& Observe Strategy.

\section{SWELL MITIGATION BY THREE-LEG DVR}

A 31\% three-phase balanced voltage swell (VS) happens at the supply side because of sudden switching a nonlinear load as shown in Figures 11a and $\mathrm{b}$. At the period $t=0.05 \mathrm{~s}$ to $0.15 \mathrm{~s}$ a three-leg DVR is connected to a distribution system and inject a compensated voltage to compensate three-phase balanced voltage swell as shown in Figure $11 \mathrm{c}$. Figure $11 \mathrm{~d}$ highlights the sinusoidal load voltage after minimizing the voltage swell effect. 


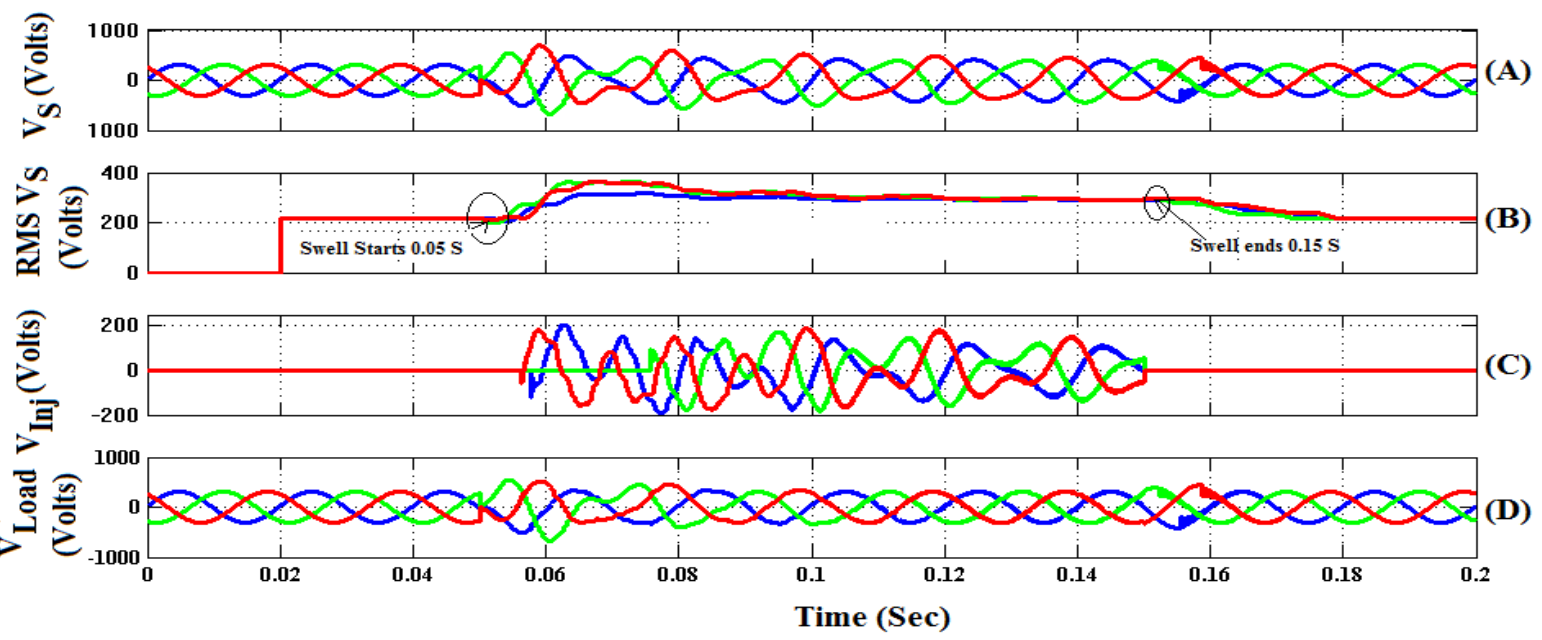

Figure 11. Swell and its mitigation by three-leg DVR.

\section{SWELL MITIGATION BY FOUR-LEG DVR}

Figures $12 a$ and $b$ show the voltage swell of magnitude $31 \%$ under sudden switching a three-phase balanced nonlinear load, which begins at $t=0.05$ second and terminates at $t=0.15$ seconds. Figure $12 c$ depicts the ability of four-leg dynamic voltage restorer to ameliorate three-phase balanced voltage swell by injecting compensating voltage. Figure $12 d$ highlights the sinusoidal load voltage after minimizing the voltage swell effect.

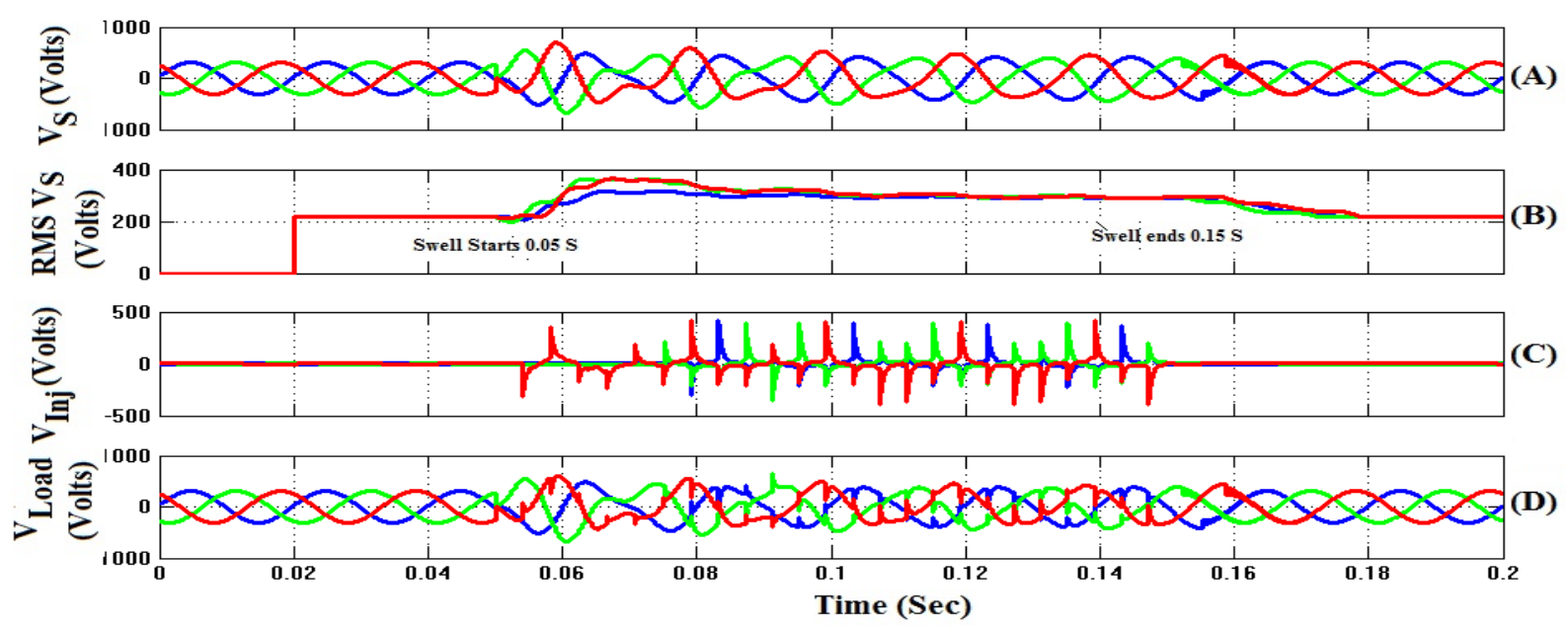

Figure 12. Voltage swell mitigation by Four-Leg DVR.

\section{SWELL MITIGATION BY SIX-LEG DVR}

Figures $13 \mathrm{a}$ and $\mathrm{b}$ shows the voltage swell of magnitude $31 \%$ under sudden switching a three-phase balanced nonlinear load in a distribution network, which begins at $\mathrm{t}=50 \mathrm{~ms}$ and terminates at $\mathrm{t}=150 \mathrm{~ms}$. Figure $13 \mathrm{c}$ depicts 
the ability of six-leg dynamic voltage restorer fed Z-source inverter to alleviate voltage swell by injecting compensating voltage. Figure $13 \mathrm{~d}$ highlights the sinusoidal load voltage after minimizing the voltage swell effect.

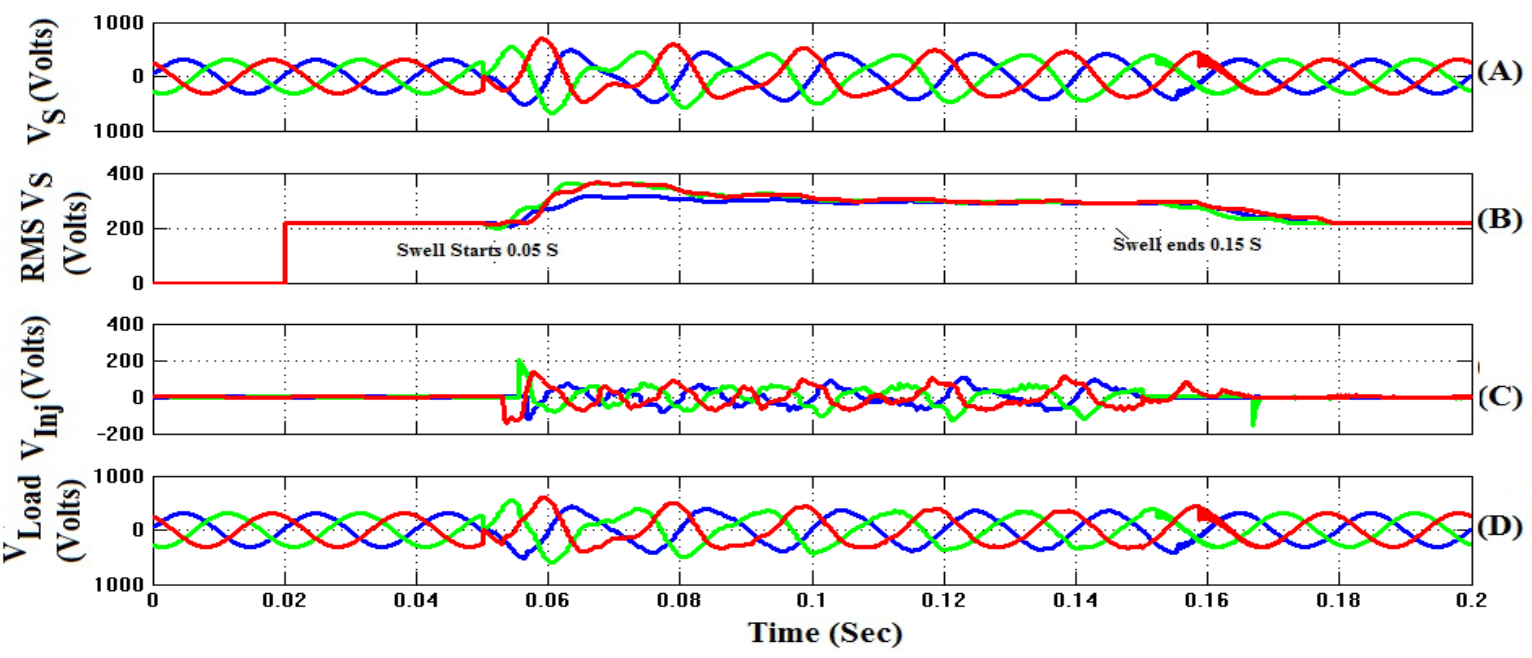

Figure 13. Voltage swell mitigation by Six-Leg DVR.

\section{PERFORMANCE COMPARISON OF THREE-LEG, FOUR-LEG AND SIX-LEG DVR}

The presentation correlation of the infused voltages of the three-leg, four-leg and six-leg DVRs are displayed in Figure 14. Because of abrupt load variation, a voltage swell of $310(98 \mathrm{~V})$ of the supply voltage is noted. The threeleg DVR comes into administration and gives the voltage of (132 V), four-leg DVR infuses the voltage of $(200 \mathrm{~V})$, and six-leg DVR infuses the voltage of $(95 \mathrm{~V})$ during voltage swell. From Figure 14, it is clearly seen that the six-leg DVR shows the better presentation to make precise missing voltage appeared differently in relation to three-leg DVR and four-leg DVR. Table 4 shows the comparison of conventional three-leg, four-leg, and six-leg VSI-DVRs with three-leg, four-leg and six-leg ZSI-DVRs.

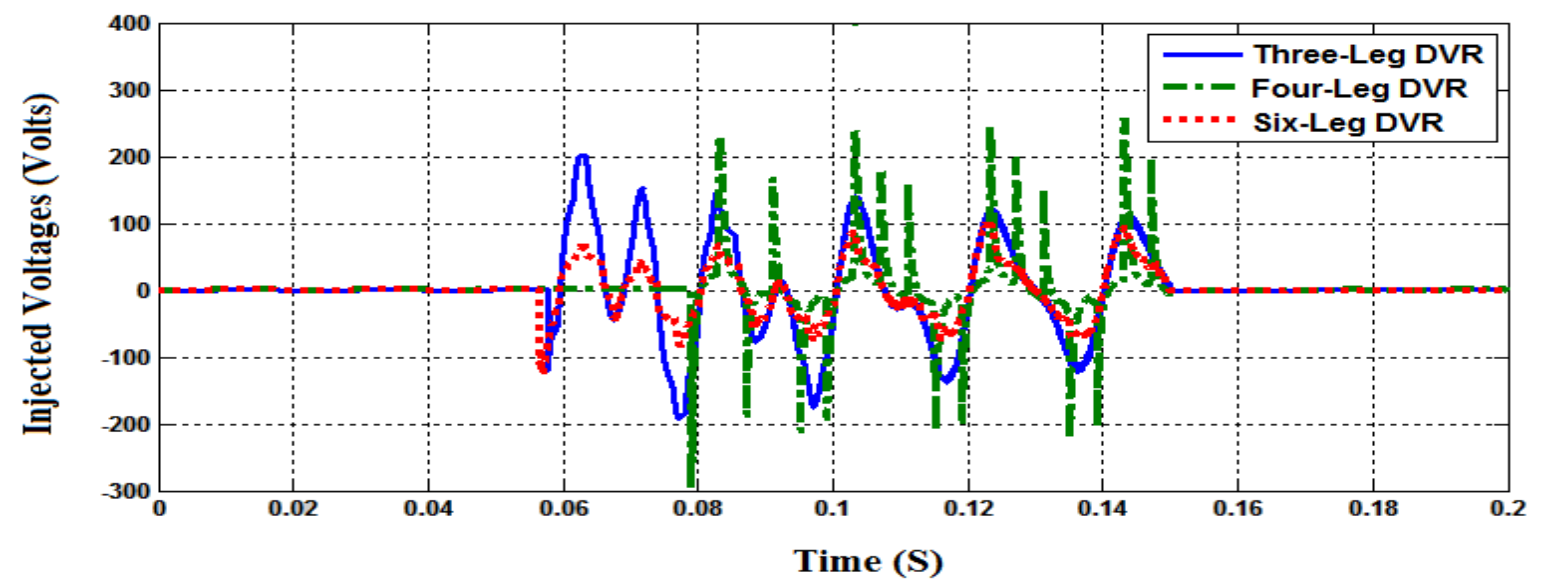

Figure 14. Comparison of injected voltages. 
Table 4. Comparison of 3-Leg, 4-Leg and 6-Leg VSI and ZSI based DVR.

\begin{tabular}{|c|c|c|c|c|c|c|}
\hline $\begin{array}{c}\text { Switching } \\
\text { States }\end{array}$ & $\begin{array}{l}\text { 3-Leg VSI-DVR } \\
\text { (Bangarraju et al., } \\
\text { 2014; Bangarraju et } \\
\text { al., 2014; Bangarraju } \\
\text { et al., 2015 ) }\end{array}$ & $\begin{array}{l}\text { 4-Leg VSI- } \\
\text { DVR } \\
\text { (Babu and } \\
\text { Ganesg } \\
\text { 2017) }\end{array}$ & $\begin{array}{l}\text { 6-Leg VSI- } \\
\text { DVR } \\
\text { (Pradeep et } \\
\text { al., 2014) }\end{array}$ & $\begin{array}{l}\text { 3-Leg ZSI- } \\
\text { DVR }\end{array}$ & $\begin{array}{l}\text { 4-Leg ZSI- } \\
\text { DVR }\end{array}$ & $\begin{array}{l}\text { 6-Leg ZSI- } \\
\text { DVR }\end{array}$ \\
\hline $\begin{array}{l}\text { Active } \\
\text { States }\end{array}$ & 6 & 14 & 40 & 6 & 14 & 40 \\
\hline $\begin{array}{l}\text { Null } \\
\text { States }\end{array}$ & 2 & 2 & 2 & 2 & 2 & 2 \\
\hline $\begin{array}{l}\text { Shoot- } \\
\text { Through } \\
\text { States }\end{array}$ & 0 & 0 & 0 & 7 & 15 & 41 \\
\hline $\begin{array}{c}\text { No of } \\
\text { Switches }\end{array}$ & 08 & 16 & 42 & 15 & 31 & 83 \\
\hline $\begin{array}{l}\text { No of } \\
\text { Legs }\end{array}$ & 3 & 4 & 6 & 3 & 4 & 6 \\
\hline $\begin{array}{l}\text { Energy } \\
\text { Stored } \\
\text { Element }\end{array}$ & Capacitor & Capacitor & Capacitor & $\begin{array}{l}\text { Capacitor } \\
\text { and } \\
\text { Inductor }\end{array}$ & $\begin{array}{l}\text { Capacitor } \\
\text { and } \\
\text { Inductor }\end{array}$ & $\begin{array}{l}\text { Capacitor } \\
\text { and } \\
\text { Inductor }\end{array}$ \\
\hline
\end{tabular}

\section{CONCLUSION}

This paper examines the solar system connected various configurations of ZSI based DVR, namely, three-phase 3-Leg, 4-Leg, and 6-Leg configurations for improving the voltage quality in the power system. For getting a maximum output power from the solar panel under change of irradiation of the sun as well as the ambient temperature of the solar panel, Perturb \& Observe (P\&O) based maximum power point tracking (MPPT) technique is used. To show the presentation examination of various configurations, the configurations are recreated in a MATLAB/SIMULINK stage. The acquired reenactment results are similar; however, they are even somewhat better in 6-leg ZSI based DVR contrasted with 3-leg and 4-leg ZSI based DVRs.

\section{REFERENCES}

Saeed, M. Q. \& Raheef, A. 2021. Adaptive-Rate Method for the Power Quality Disturbances Identification in the 5G Framework, Proceedia Computer Science, 182: 115-120

Haytham, M.A. A. Ahmed, S.A.A. Mohamed, H.A. Salama, M.M.A. 2020. Mitigating voltage-sag and voltagedeviation problems in distribution networks using battery energy storage systems, Electric Power Systems Research, 184: 106294 
Amam, H.B. Hoque, Md. Z. Li, Z. 2020. IoT Based Wireless Sensor Network for Power Quality Control in Smart Grid, Procedia Computer Science Volume, 1671148-1160

Sadigh, A.K. Smedley, K.M. 2016. Fast and precise voltage sag detection method for dynamic voltage restorer (DVR) application, Electric Power Systems Research, 130, 192-207.

Sahand, G. L. \& Mohammad, T. B. 2019. A Neural Network-Based Control Strategy for Three-Phase FourLeg Dynamic Voltage Restorer for Both Voltage Sag/Swell and Harmonic Compensation, International Power System Conference (PSC), 478-484.

Gregory, A. A. C. Cursino, B. J., Joao, P. R. A. M. Alexandre, C. O. 2017. Four-wired Dynamic Voltage Restorers Based on Cascade Open-End Winding Transformers, IEEE Energy Conversion Congress and Exposition (ECCE), 5198- 5205

Ahmed, B. El-Madjid, B. Mohamed, B. Yassine, A. Bekheira, T. 2020. Control methodology and implementation of a Z-source inverter for a stand-alone photovoltaic-diesel generator-energy storage system microgrid, Electric Power Systems Research, 185: 106-385

Taheri, A. Abbasi, B. J. Hossein B. M. 2020. LC-Z-Source Inverter Design and Control, Chinese Journal of Electronics, Vol.29, No.3, 29 (3): pp. 580-585

Tiago, M. K.F. Ronaldo, A.G. Antonio, M.S.S. 2020. Evaluation of cascaded voltage step-up cells applied to the quasi-Z-source DC-DC converter, IET, 13 (15): 3273-3282

Ali, U.S. 2018. Impedance source converter for photovoltaic stand-alone system with vanadium redox flow battery storage", Materials Today Proceedings, 5, pp. 241-247.

Rawat, M. S. and Vadhera, S. 2019. A comprehensive review on impact of wind and solar photovoltaic Energy Sources on voltage stability of power grid, Journal of Engineering Research, 7, 178-202.

Ahmed, G. Abo, K. 2020. Maximum Power Point Tracking for a PV System Using Tuned Support Vector Regression by Particle Swarm Optimization, Journal of Engineering. Research, 8 (4): 139-152

Mahiraj, S. R. and Shelly, V. 2019. A Comprehensive Review on Impact of Wind and Solar Photovoltaic Energy Sources on Voltage Stability of Power Grid, Journal of Engineering. Research, 7 (4): 178-202

Mohamed, H. Osman, M. K. Ahmed, A. R. Nikolay V. K. 2021. A Comparative Study of MPPT for PV System Based on Modified Perturbation \& Observation Method, IEEE Conference of Russian Young Researchers in Electrical and Electronic Engineering (ElConRus), 26-29 Jan. 2021, 1-6

Alik, R. and Jusoh, A. 2018. An enhanced P\&O checking algorithm MPPT for high tracking efficiency of partially shaded PV module", Solar Energy, 163, 570-580.

Jyotirmaya S. Susovon S. \& Shamik B. 2018. Adaptive PID Controller with P\&O MPPT Algorithm for Photovoltaic System, IETE Journal of Research, 66, 442-453

Bangarraju, J. Rajagopal, V. Jayalaxmi, A. 2014. Implementation of Three-Leg VSI based DVR using IRPT control algorithm, IEEE International Conference on Power Electronics (IICPE).

Bangarraju, J. Rajagopal, V. Jayalaxmi, A. 2014. Neural network control for Three-Leg VSC based DVR in Distribution System, IEEE International Conference on Power Electronics, Drives and Energy Systems,

Bangarraju, J. Rajagopal V. Nithin, B. 2015. Mitigation of supply disturbances using Three-Leg VSI based DVR from single-phase P-Q control strategy, International Conference on Electrical, Electronics, Signals, Communication and Optimization (EESCO).

Babu, G.V.S. Ganesh, V. 2017. Power quality enhancement in distributed energy resources by Four-Leg Voltage Source Converter, International Conference on Innovative Mechanisms for Industry Applications (ICIMIA), 
Pradeep, K. Niranjan, K. Akella, A.K. 2014. Six Leg DVR topology for compensation of balanced linear loads in three-phase four wire system, International Journal of System Assurrance and Engineering Management, 5, 524-533,

Sharmaa, A. Rajpurohita, B.S. Singh, S.N. 2018. A review on economics of power quality: Impact, assessment and mitigation, Renewable and Sustainable Energy Reviews, 88: 363-372 